\title{
DIVULGACIÓN CONTABLE DE INFORMACIÓN SOBRE RESPONSABILIDAD SOCIAL: ANÁLISIS COMPARATIVO DEL SECTOR FINANCIERO DE ARGENTINA Y CHILE
}

\author{
ACCOUNTING DISCLOSURE OF SOCIAL \\ RESPONSIBILITY INFORMATION: \\ A COMPARATIVE ANALYSIS OF FINANCIAL \\ SECTOR IN ARGENTINA AND CHILE
}

\author{
María de los Angeles del Barco Zillii ${ }^{1}$, María Laura Rabasedas Catalán², \\ Juan Pablo del Barco Zilli³, Sergio Miguel Hauque Fernández ${ }^{4}$
}

\section{RESUMEN}

En los últimos años, la sociedad ha incrementado su interés por el accionar de las organizaciones en materia de Responsabilidad Social Empresarial (RSE) y es por esto que las entidades buscan profundizar su gestión y resultados, mediante la publicación de memorias de sostenibilidad, informes de rendición de cuentas sociales que abarcan aspectos sobre el desempeño económico, social y ambiental organizacional. Las entidades financieras pertenecen a un sector que ha sufrido importantes cuestionamientos en materia ética y por tanto, requiere que su accionar esté asociado robustamente a conceptos de credibilidad, transparencia y responsabilidad social

En este contexto, la presente investigación tiene como objetivo determinar las características y tendencias referidas a la divulgación de información sobre responsabilidad social realizada por entidades financieras cotizadas pertenecientes a Argentina y Chile (reguladas por la CNV en Argentina y por la SVS en Chile).

Se trata de una investigación descriptiva cualitativa, realizada utilizando la técnica de análisis de contenido aplicada a las memorias de sostenibilidad publicadas por entidades financieras cotizadas para el año 2014. Sobre esta muestra, se identificaron las características de los reportes, los indicadores divulgados y se analizaron las tendencias en forma comparativa entre países.

Los resultados muestran que no existe marcada diferencia entre los dos países, destacándose la dimensión de indicadores del tipo económicos. Debido a que a la fecha no existe normativa que exija la elaboración de estos informes en ambos países, el avance en materia de RSE se muestra relativamente lento y de paulatina aplicación.

Palabras claves: Memorias de Sostenibilidad, Responsabilidad Social, Sector financiero.

Recepción: 17/01/2017. Aprobación: 11/10/2017.

1 Universidad Nacional del Litoral, Facultad de Ciencias Económicas, Santa Fe. Argentina, angelesdelbarco@hotmail.com

2 Universidad Nacional del Litoral, Facultad de Ciencias Económicas, Santa Fe. Argentina, mlrabasedas@fce.unl.edu.ar

3 Universidad Nacional del Litoral, Facultad de Ciencias Económicas, Santa Fe. Argentina, juanpablodelbarco@hotmail.com

4 Universidad Nacional del Litoral, Facultad de Ciencias Económicas, Santa Fe. Argentina, sergiohauque@yahoo.com.ar 


\begin{abstract}
In recent years, society has increased its interest on entities social responsibility (CSR) and its effects. In the seek of deepening their management results and meeting stakeholders needs, organizations develop and publish Sustainability Reports, that are accountability reports on economic, social and environmental organization performance. Accordingly, financial institutions belong to a sector that has suffered important ethical questions requering, therefore, a robus association with concepts of credibility, transparency and social responsibility.

In this context, the paper attemps to determine the characteristics and tendencies concerning the disclosure of social responsibility information by financial institutions belonging to Argentina and Chile.

To do so, we conduct a qualitative descriptive research, using content analysis on sustainability reports published by listed financial institutions in 2014. Reports characteristics, indicators disclosure and trends were determine in a comparative analysis between both countries.

The results show that there is no sustancial differences between countries, being the economic dimention the most reported set of indicators. To date, there are no regulations requiring mandatory sustainability reports, therefore progress in CSR shows relatively slow and gradual improvement and implementation.
\end{abstract}

Keywords: Financial Sector, Social Responsibility, Sustainability reports.

\section{INTRODUCCIÓN}

En los últimos años la sociedad y los diferentes stakeholders han incrementado su interés por el accionar de las organizaciones en materia de RSE al momento de definir sus criterios de inversión y sus negocios. Es por esto que las diferentes entidades buscan profundizar su gestión de responsabilidad social, exponiendo la misma a la comunidad y a sus grupos de interés.

La implementación de la cultura de RSE en las entidades colabora entre otros a la formulación de decisiones, construye fidelidad y favorece la imagen de las compañías, resultando en definitiva una mejora de la competitividad. Para evidenciar la relación entre competitividad y RSE numerosos estudios académicos han intentado relacionar la dinámica competitiva de las empresas con el "business case" de la RSE5.

El aumento de la presión competitiva y la situación económica de los mercados determinan un incremento de importancia del impacto de prácticas de responsabilidad social-iniciadas también por motivos de altruismo o éticos/religiosos (Vives, Corral e Isusi, 2006)- sobre la competitividad (Vives e Peinado Vara, 2011).

La RSE se configura así como un nuevo enfoque estratégico a la gestión de la empresa funda- do en una visión relacional de la misma (Perrini y Tencanti, 2008). La medición de la performance y la divulgación de los resultados y el accionar general de la organización a raíz de la adopción de prácticas y comportamientos, en línea con lo que dicta la responsabilidad social, asume un rol crucial (Perrini, 2006).

En materia contable y a partir del desarrollo de la contabilidad social y ambiental, la información de rendición de cuentas de las organizaciones se ha ampliado hacia aspectos referidos al desempeño económico, social y ambiental en un intento de satisfacer las necesidades y exigencias de todos los grupos de interés. En este sentido, los informes de rendición de cuentas sociales, llamados reportes de sostenibilidad o balances sociales, complementan la información que proporciona la contabilidad patrimonial y financiera tradicional (Skouloudis, 2010) sirviendo de base para la toma de decisiones de los stakeholders.

Teniendo en cuenta la importancia actual que se le otorga a la información referida al aspecto social-ambiental unido a la precariedad y/o ausencia de normas estandarizadas que unifiquen su divulgación y exposición (Muñoz Prieto, 2014), se torna necesario analizar la información que las organizaciones reportan en la actualidad.

5 Véase entre otros Thompson y Smith, 1991; Catska et al, 2004; Salzmann et al, 2005; Williamson et al, 2006; Crane et al, 2008. 
Particularmente, la presente investigación se enfoca hacia el sector financiero, debido al gran desafío al que se enfrenta luego del efecto negativo que la crisis financiera del 2008 produjo en las percepciones sociales (Matute-Vallejo et al., 2010).

Las entidades financieras requieren que su accionar esté asociado a los conceptos de credibilidad y transparencia, los cuales generan la confianza y reputación para los diferentes grupos de interés con los cuales se relacionan (Alkorta Andonegi, 2015). La concepción del comportamiento sostenible se traduce en realizar y comunicar las políticas institucionales desde la perspectiva económica, social y ambiental para con los diferentes stakeholders.

En el contexto descrito, el trabajo busca determinar las características y tendencias referidas a la divulgación de información sobre RSE del sector financiero de Argentina y Chile, como así también en forma comparativa entre ambos.

\section{METODOLOGÍA, MATERIAL Y MÉTODOS}

La presente es una investigación descriptiva cualitativa realizada en base al análisis de contenido de las memorias de sostenibilidad (MS) proporcionadas por las entidades financieras argentinas incluidas por la Comisión Nacional de Valores (CNV) como entidades emisoras a marzo 2016 y en forma comparativa con las entidades financieras chilenas incluidas por la Superintendencia de Valores y Seguros (SVS) a la misma fecha.

Para esta investigación descriptiva se emplea la técnica cualitativa de análisis de contenido (Krippendorff, 1990), la cual permite evaluar la cantidad y calidad de los datos que suministran las organizaciones (Aranguren, 2008 y Gray et al, 1996). Por otro lado, su utilidad se ha demostrado especialmente para verificar la validez en las investigaciones de presentación de información que exceden los aspectos financieros (Guthrie y Parker, 1990). (Rabasedas et al., 2016)

La muestra se acotó a las entidades bancarias cotizantes de ambos países y se compuso de un total de 26 bancos argentinos y 15 chilenos, de los cuales se procedió a realizar la búsqueda del objeto de estudio: las memorias o reportes de sostenibilidad período 2014, en las páginas web corporativas y en la base de datos del GRI.

El resultado de esta búsqueda redujo el número de la muestra a seis bancos argentinos y cinco chilenos, debido a que el resto informa actividades de RSE en su página web pero no realiza reporte de sostenibilidad y otras dos entidades (una de cada país) presentan la memoria de la compañía global la cual se encuentra presente en varios países.

Sobre esta muestra, se realizó un análisis de contenido (Krippendorff, 1990) para identificar las características de los reportes, los indicadores de sostenibilidad divulgados y las tendencias. Las variables fueron extraídas del texto de las MS y los índices de contenido GRI (ya que todos los reportes fueron realizados aplicando esta guía) luego se agruparon y analizaron en forma comparativa entre los dos países. Para cada una de estas organizaciones se analizan los principios, índices y/o indicadores económicossociales-ambientales.

\section{RESULTADOS}

Se procede a presentar los resultados en los cuales se expone en primer lugar datos generales de las MS de las entidades de la muestra para luego presentar los indicadores específicos reportados por las entidades de ambos países en forma comparativa con un resumen de la información más relevante que se obtiene de éste análisis.

\section{a) Datos generales de las memorias de soste- nibilidad y las entidades de la muestra}

Según lo informado en la tabla I, para el caso de las entidades argentinas el $80 \%$ ya se encuentra en la presentación de más de seis reportes de sostenibilidad, caso contrario para las entidades bancarias chilenas donde se puede ver que el $60 \%$ aún no ha llegado a la sexta edición de MS, siendo sólo el $40 \%$ el que supera los 9 reportes presentados ininterrumpidamente. 
Tabla I. Cantidad de reportes elaborados por las entidades bancarias desde sus orígenes.

\begin{tabular}{|c|c|c|}
\hline Cantidad reportes & Entidades argentinas & Entidades chilenas \\
\hline 0 a 3 & 0 & 2 \\
\hline Más de 3 a 6 & 1 & 1 \\
\hline Más de 6 a 9 & 4 & 0 \\
\hline Más de 9 & 1 & 2 \\
\hline
\end{tabular}

Fuente: Elaboración propia en base a datos de las MS.

\section{b) Nivel de aplicación de la guía}

Es importante destacar que todas las entidades de la muestra final presentan sus memorias aplicando la guía GRI. En cuanto al nivel de aplicación, de los seis bancos argentinos, cuatro presentan bajo la guía GRI G4, tres de ellos con la opción de criterio de conformidad "esencial" y uno "exhaustiva". Las restantes dos MS utilizaron al guía GRI G3 con los niveles $\mathrm{B}+\mathrm{y} \mathrm{C}+$.

Respecto a los bancos chilenos de los tres que presentaron bajo guía GRI G3.1, dos de ellos determinaron nivel de aplicación B y uno nivel C. En cuanto a los dos que aplicaron GRI G.4 lo hicieron con la opción de criterio de conformidad "esencial".

\section{c) Adhesión a otras normativas y principios sobre Responsabilidad Social}

Existen diferentes instrumentos a nivel internacional a implementar por las organizaciones tales como: Declaración Tripartita de OIT, OCDE, Pacto Global, ISO 26000, AA1000SES, etc.
En lo referido al análisis de la adopción de normativa/principios internacionales de RS, se obtuvo del análisis de las MS que en cuanto a las entidades argentinas el $67 \%$ adhirió a los principios del Pacto Global, una aplicó ISO 26000 y la AA1000SES03. Respecto a las entidades de Chile, el $80 \%$ expuso su alienación a los principios del Pacto Global.

\section{d) Verificación/Aseguramiento externo}

De la muestra, para los bancos argentinos el 50\% fue verificado externamente, por el contrario de los reportes chilenos se observa que ninguna entidad certificó o auditó su respectivo reporte de sostenibilidad. El aseguramiento o auditoría contribuye a la legitimidad y credibilidad de las MS ante los stakeholders a los cuales les otorga más confianza (Simnett et al., 2009; Ioannou y Serafeim, 2012). Asimismo la certificación/aseguramiento consolida el sistema de gestión interna y es garantía de cumplimiento en materia de RSE frente a los usuarios de la información.

Tabla II: Indicadores de la Guía GRI informados por las entidades bancarias de Argentina y Chile en sus Reportes de Sostenibilidad año 2014.

\begin{tabular}{|l|l|r|r|}
\hline \multicolumn{2}{|c|}{ Indicadores por categorías - Entidades bancarias } & $\begin{array}{c}\text { Present } \\
\text { Arg. } \%\end{array}$ & $\begin{array}{r}\text { Present } \\
\text { Chile } \%\end{array}$ \\
\hline 1- ECONOMÍA & 64.81 & $\mathbf{5 2 . 5 0}$ \\
\hline EC1 & 1.1. Desempeño económico & 58.33 & 70.00 \\
\hline EC2 & $\begin{array}{l}\text { Valor económico directo generado y distribuido. } \\
\text { actividades de la organización debido al cambio climático. }\end{array}$ & 83.33 & 100.00 \\
\hline EC3 & $\begin{array}{l}\text { Cobertura de las obligaciones de la organización a programas de bene- } \\
\text { ficios sociales. }\end{array}$ & 50.00 & 80.00 \\
\hline
\end{tabular}




\begin{tabular}{|c|c|c|c|}
\hline \multirow[t]{2}{*}{ EC4 } & Ayudas económicas otorgadas por entes del gobierno. & 66.66 & 80.00 \\
\hline & 1.2. Presencia en el mercado & 83.33 & 60.00 \\
\hline EC5 & $\begin{array}{l}\text { Relación entre el salario inicial y el salario mínimo local (desglosado } \\
\text { por sexo). }\end{array}$ & 83.33 & 80.00 \\
\hline \multirow[t]{2}{*}{ EC6 } & $\%$ de altos directivos procedentes de la comunidad local. & 83.33 & 40.00 \\
\hline & 1.3. Consecuencias económicas directas & 66.66 & 40.00 \\
\hline EC7 & $\begin{array}{l}\text { Desarrollo e impacto de la inversión en infraestructuras y los tipos de } \\
\text { servicios. }\end{array}$ & 66.66 & 20.00 \\
\hline \multirow[t]{2}{*}{ EC8 } & Impactos económicos indirectos significativos y alcance de los mismos. & 66.66 & 60.00 \\
\hline & 1.4. Prácticas de adquisición & 40.00 & 50.00 \\
\hline EC9 & $\begin{array}{l}\% \text { del gasto en los lugares con operaciones significativas con proveedo- } \\
\text { res locales. }\end{array}$ & 40.00 & 50.00 \\
\hline \multicolumn{2}{|r|}{ 2- MEDIO AMBIENTE } & 37.40 & 30.49 \\
\hline & 2.1. Materiales & 58.33 & 43.33 \\
\hline EN1 & Materiales por peso o volumen. & 66.66 & 60.00 \\
\hline \multirow[t]{2}{*}{ EN2 } & $\%$ de los materiales utilizados que son materiales reciclados. & 50.00 & 40.00 \\
\hline & 2.2. Energía & 63.32 & 44.00 \\
\hline EN3 & Consumo energético interno. & 83.33 & 60.00 \\
\hline EN4 & Consumo energético externo. & 33.33 & 40.00 \\
\hline EN5 & Intensidad energética. & 66.66 & 40.00 \\
\hline EN6 & Reducción del consumo energético. & 66.66 & 40.00 \\
\hline \multirow[t]{2}{*}{ EN7 } & Reducciones de los requisitos energéticos de los productos y servicios. & 66.66 & 40.00 \\
\hline & 2.3. Agua & 38.88 & 46.67 \\
\hline EN8 & Captación total de agua por fuente. & 50.00 & 60.00 \\
\hline EN9 & Fuentes de agua afectadas significativamente por la captación de agua. & 50.00 & 40.00 \\
\hline \multirow[t]{2}{*}{ EN10 } & $\%$ y volumen total de agua reciclada y reutilizada. & 16.66 & 40.00 \\
\hline & 2.4. Biodiversidad & 20.00 & 16.66 \\
\hline EN11 & $\begin{array}{l}\text { Instalaciones operativas que sean adyacentes, contengan o estén ubica- } \\
\text { das en áreas protegidas y no protegidas de gran valor para la biodiver- } \\
\text { sidad. }\end{array}$ & 20.00 & 16.66 \\
\hline EN12 & $\begin{array}{l}\text { Impactos más significativos en la biodiversidad de áreas protegidas o } \\
\text { áreas de alta biodiversidad no protegidas, derivados de las actividades, } \\
\text { productos y servicios. }\end{array}$ & 20.00 & 16.66 \\
\hline EN13 & Hábitats protegidos o restaurados. & 20.00 & 16.66 \\
\hline \multirow[t]{2}{*}{ EN14 } & $\begin{array}{l}\text { Número de especies cuyos hábitats se encuentran en áreas afectadas } \\
\text { por las operaciones. }\end{array}$ & 20.00 & 16.66 \\
\hline & 2.5. Emisiones & 40.00 & 38.09 \\
\hline EN15 & Emisiones directas de gases de efecto invernadero. & 60.00 & 50.00 \\
\hline EN16 & Emisiones indirectas de gases de efecto invernadero al generar energía. & 40.00 & 50.00 \\
\hline EN17 & Otras emisiones indirectas de gases de efecto invernadero. & 40.00 & 33.33 \\
\hline EN18 & Intensidad de las emisiones de gases de efecto invernadero. & 20.00 & 16.66 \\
\hline EN19 & Reducción de las emisiones de gases de efecto invernadero. & 40.00 & 33.33 \\
\hline
\end{tabular}




\begin{tabular}{|c|c|c|c|}
\hline EN20 & Emisiones de sustancias que agotan el ozono. & 40.00 & 50.00 \\
\hline \multirow[t]{2}{*}{ EN21 } & NOX, SOX Y Otras emisiones atmosféricas significativas. & 40.00 & 33.33 \\
\hline & 2.6. Efluentes y Residuos & 28.00 & 43.33 \\
\hline EN22 & Vertido total de aguas, según su calidad y destino. & 40.00 & 33.33 \\
\hline EN23 & Peso total de los residuos, según tipo y método de tratamiento. & 40.00 & 50.00 \\
\hline EN24 & Número y volumen totales de los derrames significativos. & 20.00 & 50.00 \\
\hline EN25 & $\begin{array}{l}\text { Peso de los residuos transportados, importados, exportados o tratados } \\
\text { que se consideran peligrosos y porcentaje de residuos transportados } \\
\text { internacionalmente. }\end{array}$ & 20.00 & 33.33 \\
\hline \multirow[t]{2}{*}{ EN26 } & $\begin{array}{l}\text { Identificación, tamaño, estado de protección y valor de biodiversidad } \\
\text { de las masas de agua y los hábitats relacionados afectados significativa- } \\
\text { mente por vertidos de la org. }\end{array}$ & 20.00 & 50.00 \\
\hline & 2.7. Productos y servicios & 44.44 & 46.67 \\
\hline EN27 & $\begin{array}{l}\text { Grado de mitigación del impacto ambiental de los productos y servi- } \\
\text { cios. }\end{array}$ & 50.00 & 40.00 \\
\hline \multirow[t]{2}{*}{ EN28 } & $\begin{array}{l}\text { \% de los productos vendidos y sus materiales de embalaje que se recu- } \\
\text { peran al final de su vida útil, por categorías de productos. }\end{array}$ & 50.00 & 60.00 \\
\hline & Cumplimiento regulatorio. & & \\
\hline \multirow[t]{2}{*}{ EN29 } & $\begin{array}{l}\text { Valor monetario de las multas significativas y número de sanciones no } \\
\text { monetarias por incumplimiento de la legislación y la normativa am- } \\
\text { biental. }\end{array}$ & 33.33 & 40.00 \\
\hline & 2.8. Transporte & 20.00 & 33.00 \\
\hline \multirow[t]{2}{*}{ EN30 } & $\begin{array}{l}\text { Impactos ambientales significativos del transporte de productos y otros } \\
\text { bienes y materiales utilizados, así como del transporte de personal. }\end{array}$ & 20.00 & 33.00 \\
\hline & 2.9. General & 0.00 & 0.00 \\
\hline \multirow[t]{2}{*}{ EN31 } & Desglose de los gastos y las inversiones ambientales. & 0 & 0 \\
\hline & 2.10. Evaluación ambiental de los proveedores & 20.00 & 25.00 \\
\hline EN32 & $\begin{array}{l}\% \text { de nuevos proveedores examinados en función de criterios ambien- } \\
\text { tales. }\end{array}$ & 20.00 & 33.33 \\
\hline \multirow[t]{2}{*}{ EN33 } & Impactos ambientales negativos significativos, reales y potenciales. & 20.00 & 16.66 \\
\hline & 2.11. Mecanismos de reclamación ambiental & 20.00 & 33.33 \\
\hline EN34 & $\begin{array}{l}\text { Número de reclamaciones ambientales presentadas, abordado y resul- } \\
\text { tado. }\end{array}$ & 20.00 & 33.33 \\
\hline \multicolumn{2}{|r|}{ 3. DESEMPEÑO SOCIAL } & 50.80 & 52.06 \\
\hline \multicolumn{2}{|r|}{ 3.1.PRÁCTICAS LABORALES Y TRABAJO DIGNO } & 55.90 & 54.16 \\
\hline & 3.1.1. Empleo & 60.00 & 61.11 \\
\hline LA1 & Número y tasa de contrataciones y rotación media de empleados. & 100.00 & 83.33 \\
\hline LA2 & $\begin{array}{l}\text { Prestaciones sociales para los empleados de jornada completa que no } \\
\text { se ofrecen a empleados temporales o de media jornada. }\end{array}$ & 60.00 & 83.33 \\
\hline \multirow[t]{2}{*}{ LA3 } & $\begin{array}{l}\text { Índices de reincorporación y de retención tras la licencia por } \\
\text { maternidad o paternidad. }\end{array}$ & 20.00 & 16.66 \\
\hline & 3.1.2. Relaciones entre los trabajadores y la dirección & 60.00 & 50.00 \\
\hline LA4 & $\begin{array}{l}\text { Plazos mínimos de preaviso de cambios operativos y posible inclusión } \\
\text { en los CCT. }\end{array}$ & 60.00 & 50.00 \\
\hline
\end{tabular}




\begin{tabular}{|c|c|c|c|}
\hline & 3.1.3. Salud y seguridad en el trabajo & 60.00 & 58.33 \\
\hline LA5 & $\begin{array}{l}\text { \% de trabajadores que está representado en comités formales de salud } \\
\text { y seguridad conjuntos para la dirección y empleados, establecidos para } \\
\text { ayudar a controlar y asesorar sobre programas de salud y seguridad en } \\
\text { el trabajo. }\end{array}$ & 80.00 & 50.00 \\
\hline LA6 & $\begin{array}{l}\text { Tipo y tasa de lesiones, enfermedades profesionales, días perdidos, } \\
\text { ausentismo y número de víctimas mortales relacionadas con el trabajo. }\end{array}$ & 40.00 & 66.66 \\
\hline LA7 & $\begin{array}{l}\text { Trabajadores cuya profesión tiene una incidencia o un riesgo elevado } \\
\text { de enfermedad. }\end{array}$ & 40.00 & 33.33 \\
\hline \multirow[t]{2}{*}{ LA8 } & $\begin{array}{l}\text { Asuntos de salud y seguridad cubiertos en acuerdos formales con los } \\
\text { sindicatos. }\end{array}$ & 80.00 & 83.33 \\
\hline & 3.1.4. Capacitación y educación & 93.33 & 94.44 \\
\hline LA9 & Promedio de horas de capacitación anuales por empleado. & 100.00 & 100.00 \\
\hline LA10 & $\begin{array}{l}\text { Programas de gestión de habilidades y de formación continua que fo- } \\
\text { mentan la empleabilidad de los trabajadores y les ayuda a finalizar sus } \\
\text { carreras profesionales. }\end{array}$ & 100.00 & 83.33 \\
\hline \multirow[t]{2}{*}{ LA11 } & $\begin{array}{l}\% \text { de empleados que reciben evaluaciones regulares del desempeño y } \\
\text { de desarrollo profesional. }\end{array}$ & 80.00 & 100.00 \\
\hline & 3.1.5. Diversidad e igualdad de oportunidades & 60.00 & 83.33 \\
\hline \multirow[t]{2}{*}{ LA12 } & $\begin{array}{l}\text { Composición de los órganos de gobierno y desglose de la plantilla por } \\
\text { categoría profesional y sexo, edad, pertenencia a minorías y otros indi- } \\
\text { cadores de diversidad. }\end{array}$ & 60.00 & 83.33 \\
\hline & 3.1.6. Igualdad de retribución entre mujeres y hombres & 60.00 & 50.00 \\
\hline \multirow[t]{2}{*}{ LA13 } & $\begin{array}{l}\text { Relación entre el salario base de los hombres con respecto al de las } \\
\text { mujeres. }\end{array}$ & 60.00 & 50.00 \\
\hline & 3.1.7. Evaluación de las prácticas laborales de los proveedores & 20.00 & 16.66 \\
\hline LA14 & $\begin{array}{l}\% \text { de nuevos proveedores examinados en función de criterios relativos } \\
\text { a las prácticas laborales. }\end{array}$ & 20.00 & 16.66 \\
\hline \multirow[t]{2}{*}{ LA15 } & $\begin{array}{l}\text { Impactos negativos significativos, reales y potenciales, en las prácticas } \\
\text { laborales en la cadena de suministro, y las medidas al respecto. }\end{array}$ & 20.00 & 16.66 \\
\hline & 3.1.8. Mecanismos de reclamo sobre las prácticas laborales & 20.00 & 33.33 \\
\hline LA16 & $\begin{array}{l}\mathrm{N}^{o} \text { de reclamaciones sobre prácticas laborales que se han presentado, } \\
\text { abordado y resuelto. }\end{array}$ & 20.00 & 33.33 \\
\hline \multicolumn{2}{|r|}{ 3.2. DERECHOS HUMANOS } & 37.03 & 41.00 \\
\hline & 3.2.1. Inversión & 33.33 & 70.00 \\
\hline HR1 & $\begin{array}{l}\text { Número y \% de contratos y acuerdos de inversión significativos que } \\
\text { incluyen cláusulas de derechos humanos o que han sido objeto de aná- } \\
\text { lisis en materia de DDHH. }\end{array}$ & 16.66 & 60.00 \\
\hline \multirow[t]{2}{*}{ HR2 } & $\begin{array}{l}\text { Hs. de formación de los empleados s / políticas y procedimientos rela- } \\
\text { cionados con DDHH. }\end{array}$ & 50.00 & 80.00 \\
\hline & 3.2.2. No discriminación & 50.00 & 80.00 \\
\hline \multirow[t]{2}{*}{ HR3 } & Número de casos de discriminación y medidas correctivas adoptadas. & 50.00 & 80.00 \\
\hline & 3.2.3. Libertad de asociación y negociación colectiva & 50.00 & 60.00 \\
\hline HR4 & $\begin{array}{l}\text { Identificación de centros y proveedores significativos en los que la li- } \\
\text { bertad de asociación a convenios colectivos pueden infringirse o estar } \\
\text { amenazados y medidas adoptadas para defender estos derechos. }\end{array}$ & 50.00 & 60.00 \\
\hline
\end{tabular}




\begin{tabular}{|c|c|c|c|}
\hline & 3.2.4. Trabajo infantil & 50.00 & 40.00 \\
\hline \multirow[t]{2}{*}{ HR5 } & $\begin{array}{l}\text { Identificación de centros y proveedores con un riesgo significativos de } \\
\text { casos de explotación infantil y medidas adoptadas para contribuir a la } \\
\text { abolición de la explotación infantil. }\end{array}$ & 50.00 & 40.00 \\
\hline & 3.2.5. Trabajo forzoso & 33.33 & 40.00 \\
\hline \multirow[t]{2}{*}{ HR6 } & $\begin{array}{l}\text { Centros y proveedores con un riesgo significativo de ser origen de } \\
\text { episodios de trabajo forzoso y medidas adoptadas para contribuir a la } \\
\text { eliminación de trabajo forzoso. }\end{array}$ & 33.33 & 40.00 \\
\hline & 3.2.6. Medidas de seguridad & 16.66 & 20.00 \\
\hline \multirow[t]{2}{*}{ HR7 } & $\begin{array}{l}\% \text { del personal de seguridad que ha recibido capacitación sobre las } \\
\text { políticas o procedimientos en materia de derechos humanos relevantes } \\
\text { para las operaciones. }\end{array}$ & 16.66 & 20.00 \\
\hline & 3.2.7. Derechos de la población indígena & 33.33 & 20.00 \\
\hline \multirow[t]{2}{*}{ HR8 } & $\begin{array}{l}\mathrm{N}^{\circ} \text { de casos de violación de los derechos de los pueblos indígenas y } \\
\text { medidas adoptadas. }\end{array}$ & 33.33 & 20.00 \\
\hline & 3.2.8. Evaluación & 33.33 & 20.00 \\
\hline \multirow[t]{2}{*}{ HR9 } & $\begin{array}{l}N^{\circ} \text { y } \% \text { de centros objeto de exámenes o evaluaciones de impactos en } \\
\text { DDHH. }\end{array}$ & 33.33 & 20.00 \\
\hline & 3.2.9. Evaluación de los proveedores en materia de derechos humanos & 33.33 & 40.00 \\
\hline HR10 & $\begin{array}{l}\% \text { de nuevos proveedores examinados en función de criterios relativos } \\
\text { a los DDHH. }\end{array}$ & 50.00 & 60.00 \\
\hline \multirow[t]{2}{*}{ HR11 } & $\begin{array}{l}\text { Impactos negativos significativos en materia de DDHH, reales y poten- } \\
\text { ciales, en la cadena de suministro y las medidas adoptadas. }\end{array}$ & 16.66 & 20.00 \\
\hline & 3.2.10. Mecanismos de reclamo en materia de derechos humanos & 0.00 & 20.00 \\
\hline HR12 & $\begin{array}{l}\mathrm{N}^{o} \text { de reclamaciones sobre DDHH que se han presentado, abordado y } \\
\text { resuelto. }\end{array}$ & 0.00 & 20.00 \\
\hline \multicolumn{2}{|c|}{ 3.3. SOCIEDAD } & 42.38 & 50.95 \\
\hline & 3.3.1. Comunidades locales & 50.00 & 50.00 \\
\hline SO1 & $\begin{array}{l}\% \text { de centros donde se han implantado programas de desarrollo, eva- } \\
\text { luaciones de impacto y participación de la comunidad local. }\end{array}$ & 83.33 & 83.33 \\
\hline \multirow[t]{2}{*}{$\mathrm{SO} 2$} & $\begin{array}{l}\text { Centros de operaciones con efectos negativos significativos, reales o } \\
\text { potenciales, sobre las comunidades locales. }\end{array}$ & 16.66 & 16.66 \\
\hline & 3.3.2. Lucha contra la corrupción & 66.66 & 66.66 \\
\hline SO3 & $\begin{array}{l}\text { Número y \% de centros en los que se han evaluado los riesgos relacio- } \\
\text { nados con la corrupción y riesgos significativos detectados. }\end{array}$ & 66.66 & 40.00 \\
\hline $\mathrm{SO} 4$ & $\begin{array}{l}\text { Políticas y procedimientos de comunicación y capacitación s/lucha } \\
\text { contra la corrupción. }\end{array}$ & 83.33 & 80.00 \\
\hline \multirow[t]{2}{*}{$\mathrm{SO} 5$} & Casos confirmados de corrupción y medidas adoptadas. & 50.00 & 80.00 \\
\hline & 3.3.3. Política pública & 33.33 & 60.00 \\
\hline \multirow[t]{2}{*}{ SO6 } & Valor de las contribuciones políticas, por país y destinatario. & 16.66 & 60.00 \\
\hline & 3.3.4. Prácticas de competencia desleal & 50.00 & 60.00 \\
\hline \multirow[t]{2}{*}{ SO7 } & $\begin{array}{l}\text { Número de demandas por competencia desleal, prácticas monopólicas } \\
\text { o contra la libre competencia y resultado de las mismas. }\end{array}$ & 50.00 & 60.00 \\
\hline & 3.3.5. Cumplimiento regulatorio & 50.00 & 60.00 \\
\hline
\end{tabular}




\begin{tabular}{|c|c|c|c|}
\hline $\mathrm{SO} 8$ & $\begin{array}{l}\text { Valor monetario de las multas significativas y número de sanciones no } \\
\text { monetarias por incumplimiento de la legislación y la normativa. }\end{array}$ & 50.00 & 60.00 \\
\hline & 3.3.6. Evaluación de la repercusión social de los proveedores & 0.00 & 20.00 \\
\hline SO9 & $\begin{array}{l}\text { \% de nuevos proveedores que se examinaron en función de criterios } \\
\text { relacionados con la repercusión social. }\end{array}$ & 0.00 & 20.00 \\
\hline \multirow[t]{2}{*}{ SO10 } & $\begin{array}{l}\text { Impactos negativos significativos y potenciales para la sociedad en la } \\
\text { cadena de suministro y medidas adoptadas. }\end{array}$ & 0.00 & 20.00 \\
\hline & 3.3.7. Mecanismos de reclamación por impacto social & 0.00 & 20.00 \\
\hline SO11 & $\begin{array}{l}\mathrm{N}^{\mathrm{o}} \text { de reclamaciones sobre impactos sociales presentadas, abordadas y } \\
\text { resueltas. }\end{array}$ & 0.00 & 20.00 \\
\hline \multicolumn{2}{|r|}{ 3.4. RESPONSABILIDAD SOBRE LOS PRODUCTOS } & 65.83 & 73.50 \\
\hline & 3.4.1 Salud y seguridad de los clientes & 64.00 & 63.33 \\
\hline PR1 & $\begin{array}{l}\text { \% de categorías de productos y servicios significativos cuyos impactos } \\
\text { en materia de salud y seguridad se han evaluado para promover mejo- } \\
\text { ras. }\end{array}$ & 20.00 & 50.00 \\
\hline PR2 & $\begin{array}{l}\mathrm{N}^{\circ} \text { de incidentes por incumplimiento de la normativa o de los códigos } \\
\text { voluntarios relativos a los impactos de los productos y servicios en la } \\
\text { salud y la seguridad durante su ciclo de vida. }\end{array}$ & 40.00 & 66.66 \\
\hline PR3 & $\begin{array}{l}\text { Tipo de información que requieren los procedimientos de la organiza- } \\
\text { ción relativos a la información y el etiquetado de sus productos y ser- } \\
\text { vicios y porcentaje de categorías de productos y servicios significativos } \\
\text { que están sujetas a tales requisitos. }\end{array}$ & 100.00 & 50.00 \\
\hline PR4 & $\begin{array}{l}\text { Número de incumplimientos de la regulación y de los códigos volun- } \\
\text { tarios relativos a la información y al etiquetado de los productos y } \\
\text { servicios. }\end{array}$ & 60.00 & 66.66 \\
\hline \multirow[t]{2}{*}{ PR5 } & Resultados de las encuestas para medir la satisfacción de los clientes & 100.00 & 83.33 \\
\hline & 3.4.2. Comunicaciones de mercadotecnia & 70.00 & 50.00 \\
\hline PR6 & Ventas de productos prohibidos o en litigio. & 60.00 & 50.00 \\
\hline \multirow[t]{2}{*}{ PR7 } & $\begin{array}{l}N^{\circ} \text { de casos de incumplimientos de la normativa o los códigos volun- } \\
\text { tarios relativos a las comunicaciones de mercadotecnia, tales como la } \\
\text { publicidad, la promoción y el patrocinio. }\end{array}$ & 80.00 & 50.00 \\
\hline & 3.4.3. Privacidad de los clientes & 80.00 & 83.33 \\
\hline \multirow[t]{2}{*}{ PR8 } & $\begin{array}{l}N^{\circ} \text { de reclamos s/la violación de la privacidad y la fuga de datos de los } \\
\text { clientes. }\end{array}$ & 80.00 & 83.33 \\
\hline & 3.4.4. Cumplimiento regulatorio & 80.00 & 66.66 \\
\hline PR9 & $\begin{array}{l}\text { Costo de las multas significativas por incumplir la normativa y la legis- } \\
\text { lación relativas al suministro y el uso de productos y servicios. }\end{array}$ & 65.83 & 66.66 \\
\hline
\end{tabular}

Fuente: Elaboración propia en base a datos de las MS.

Como se puede observar en la tabla II, las entidades bancarias argentinas de las tres categorías que presenta la guía GRI para los reportes de sostenibilidad, informan en mayor porcentaje la categoría economía $(64.81 \%)$, luego desempeño social $(50.80 \%)$ y en menor frecuencia sobre medio ambiente $(37.40 \%)$.
Dentro de la categoría economía, se informa con mayor frecuencia aspectos relacionados con la presencia en el mercado y como indicadores más reportados: Valor económico generado y distribuido (EC1), Relación entre salario inicial y salario mínimo local (EC5) y porcentaje de directores locales (EC6). El indicador menos informa- 
do es el EC3 referido a la cobertura de las obligaciones de la organización debidas a programas de beneficios sociales.

Respecto a la segunda categoría más reportada, desempeño social, la que mayor cantidad de entidades informa responsabilidad sobre productos $(65,83 \%)$, prácticas laborales y trabajo digno $(55,90 \%)$, sociedad $(42,38 \%)$ y por último derechos humanos (37,03\%). En la subcategoría de responsabilidad sobre los productos el aspecto más informado es privacidad de los clientes, enumerando la cantidad de reclamos por violación de privacidad y fuga de datos. Con el igual porcentaje de presentación se muestran los resultados de las encuestas de satisfacción de los clientes. Esto reafirma la concepción que éste tipo de información es de vital importancia para las entidades bancarias, ya que los datos de clientes y la atención son puntos claves del servicio y determinan la reputación bancaria.

En la subcategoría prácticas laborales y trabajo digno, el aspecto más informado no sólo de ésta sino respecto de la totalidad de los aspectos de la guía GRI, es el de capacitación y educación, donde se puede ver como el $100 \%$ reporta información sobre horas de capacitación por empleado (LA9) y evaluaciones de desempeño y desarrollo profesional (LA12). En esta subcategoría los indicadores menos reportados fueron los índices de reincorporación al trabajo al cabo del goce de licencias por maternidad o paternidad (LA3), y los indicadores LA14 y LA15 que corresponden a evaluación de prácticas laborales de los proveedores.

Tal como se muestra en la tabla II, en sociedad, el aspecto referido a la lucha contra la corrupción es el más informado, seguido por el indicador sobre políticas y procedimientos de comunicación y capacitación y el porcentaje de centros donde se han implantado programas de desarrollo y participación de la comunidad local. Cabe destacar que ninguna entidad hizo mención sobre la evaluación de la repercusión social de los proveedores y los mecanismos de reclamación por impacto social

Como última subcategoría y con menor frecuencia de presentación se encuentran los derechos humanos en la cual los aspectos de libertad de asociación y negociación colectiva, trabajo infantil y no discriminación son reportados por el $50 \%$ de la muestra.

Respecto a la categoría medio ambiente, las entidades bancarias reportan en mayor proporción los indicadores referidos a energía, siendo el EN3 sobre consumo energético interno, el más informado. Se considera que el consumo de energía en las entidades bancarias es el que más incidencia tiene sobre la actividad que desarrollan, hecho que se comprueba con el reporte de este indicador.

Continuando el análisis, ahora para las entidades chilenas de la muestra, se puede señalar que el principal aspecto informado es el económico $(52.50 \%)$, seguido por una ínfima diferencia por el aspecto de desempeño social $(52.06 \%)$ y por último medio ambiente $(30.49 \%)$.

En la categoría de economía, el aspecto más reportado es el de desempeño económico (70\%) seguido por presencia en el mercado $(60 \%)$. En referencia a los indicadores principales se puede observar que la totalidad de las entidades informan el Estado de valor económico generado y distribuido (EC1) y como indicador con menor frecuencia el que refiere a impacto de inversión en infraestructura y tipos de servicios.

En desempeño social, se observa en cuanto a cantidad de indicadores reportados con: Responsabilidad sobre los productos $(73.50 \%)$, prácticas laborales y trabajo digno $(54.16 \%)$, sociedad $(50.95 \%)$ y derechos humanos $(41 \%)$. De todas las subcategorías, la totalidad de las empresas informan sobre los indicadores de cantidad de contrataciones y rotación de empleados (LA1), horas promedio de capacitación de los mismos (LA9), programas de gestión de habilidades y formación continua (LA10), centros de programas de desarrollo y participación de la comunidad local (SO1), procedimientos de la organización respecto a datos sobre servicios (PR3), Resultados de las encuestas sobre satisfacción de los clientes (PR5).

Para la categoría de medio ambiente los aspectos más reportados fueron agua $(46.67 \%)$ y productos y servicios $(46.67 \%)$ seguidos por energía $(44 \%)$. Como indicadores presentados por más de la mitad de las empresas de la muestra se encuentran el EN3 sobre consumo energético interno, EN8 captación total de agua por fuente, EN1 materiales por peso o volumen y el EN28 sobre materiales o productos que se recuperan al término de su vida útil.

Realizando un análisis comparativo de los aspectos más reportados tanto por las entidades argentinas como chilenas de la muestra, según se puede ver en el Gráfico 1, en ambos casos la categoría más reportada es la económica, seguida por sociedad y por último medio ambiente. 


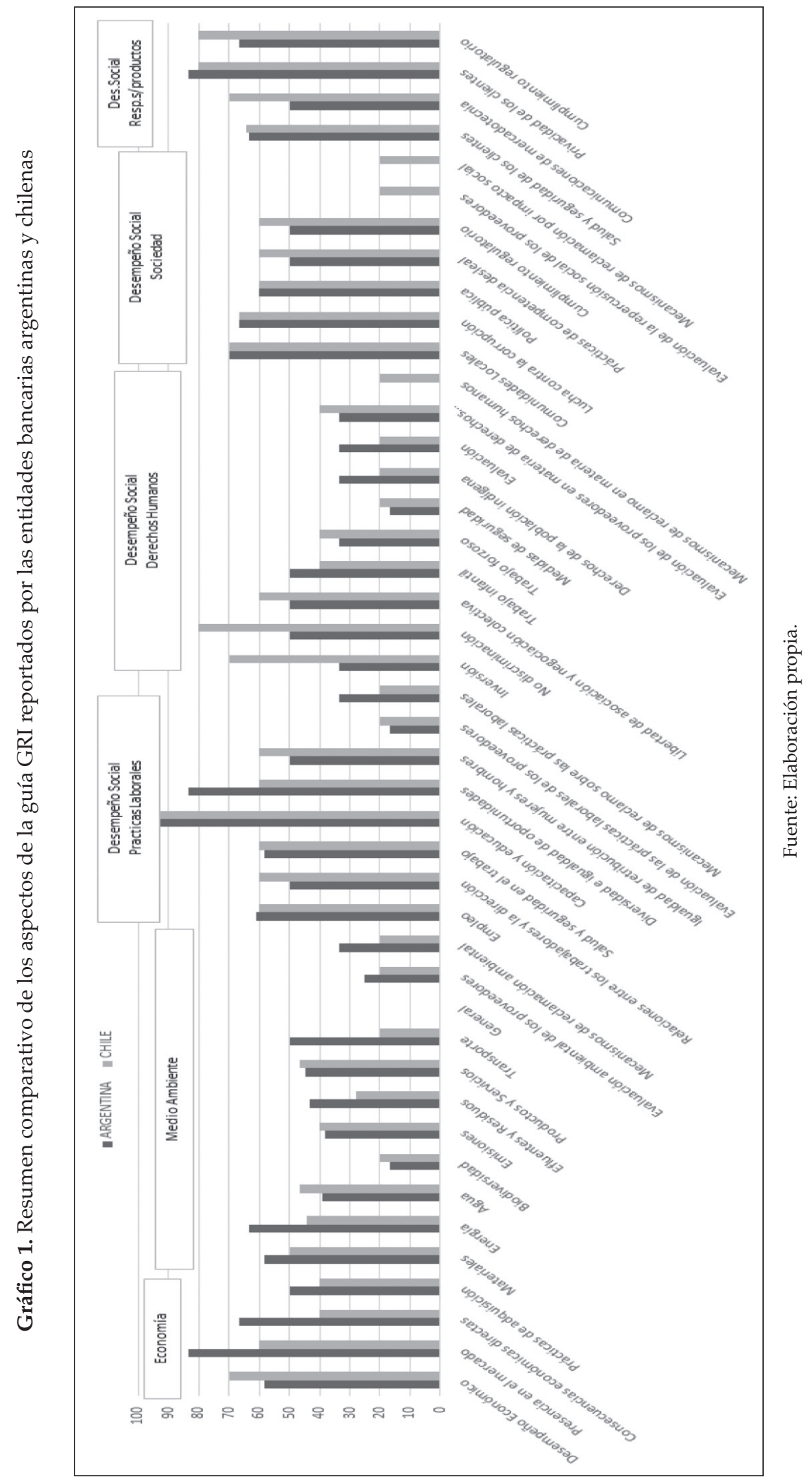


En cuanto al reporte del suplemento sectorial para servicios financieros, de las seis entidades argentinas de la muestra solamente tres lo presentan, de las cuales la que mayor cantidad de indicadores informa son once y la que menos son cuatro indicadores. Se observa en el Gráfico
2 que la totalidad de los bancos argentinos que informan este complemento presentan datos sobre la educación financiera e iniciativas para mejorar el acceso a los servicios por parte de personas menos favorecidas.

Gráfico 2. Indicadores del suplemento sectorial para servicios financieros reportados por las entidades.

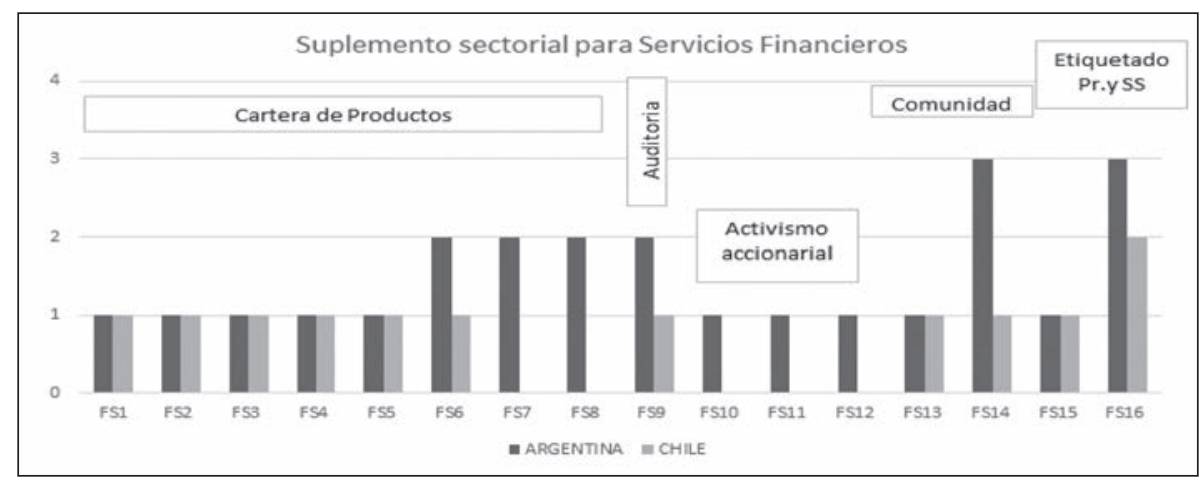

Fuente: Elaboración propia en base a datos de las MS.

Respecto al suplemento para el sector financiero presentado por las entidades bancarias chilenas, cuatro de las cinco entidades de la muestra reportan información sobre algunos de los indicadores del suplemento. Como demuestra el Gráfico 2, las cuatro entidades seleccionan diferentes indicadores del suplemento para su inclusión en el reporte de sostenibilidad. La entidad bancaria que más indicadores reportó seleccionó cinco indicadores y la que menos reportó un solo indicador. Por lo cual, entendemos que a pesar de la presentación de este suplemento, en general no se brinda más información que la de los indicadores generales.

\section{CONCLUSIÓN}

A modo de conclusión, es posible afirmar que, de acuerdo a los resultados obtenidos, no existe amplia diferencia en cuanto al tipo de información que las entidades financieras de ambos países brindan en sus memorias de sostenibilidad, siendo ésta escasa y no muy precisa e integral. Debido a que a la fecha no existe normativa que exija la elaboración de estos informes en ambos países, esto conlleva a que el avance en materia de RSE sea lento y de paulatina aplicación.
La realización de estos informes de sostenibilidad tiene sobre todo un impacto interno para la organización, en términos de autoanálisis. En tal sentido representa un momento de reflexión sobre los compromisos y el grado de cumplimiento de los objetivos prefijados en las diversas áreas de gestión y en las interacciones con los stakeholders. También permite demostrar al externo de la organización la capacidad de crear y distribuir el valor, reforzando los vínculos entre los distintos interlocutores de la misma (Hauque et al., 2016).

Las entidades financieras al pertenecer a un sector relevante de la comunidad en los últimos años ha sufrido importantes crisis (basadas sobretodo en cuestiones éticas), deberían adaptar su estrategia de negocio centrándola en la responsabilidad social y rendir cuentas a los diferentes grupos de interés a través de las herramientas actuales como son los informes de sostenibilidad.

Cada vez son más las organizaciones, independientemente del sector analizado, que se interesan en la problemática social, ambiental y económica, ya que todo indica que el desarrollo de la RSE mejora sustancialmente su reputación y legitimidad (Kabir, 2011; Lindorff et al., 2012). Asimismo existe una presión actual constante de los grupos de interés por exigir a las organiza- 
ciones prácticas en RSE y su divulgación, esto no sólo beneficia a los stakeholders sino que permite generar una mayor confianza entre ellos y las entidades y de ésta forma lograr el éxito esperado (Font et al., 2012).

Un factor que influye en la presentación voluntaria de los informes de sostenibilidad, son los costos de realización de los mismos (gastos de recopilación de datos, procesamiento y publicación, dificultades de medición y verificación de los aspectos socio-ambientales, etc.) (Ahmad et al., 2003). No todas las entidades logran realizarlos e incluso, algunas sólo informan acciones aisladas de acuerdo al análisis propio de coste / beneficio (Brammer y Pavelin, 2004).

Se entiende que pese al crecimiento actual en la divulgación de informes de sostenibilidad, siguen existiendo cuestionamientos y aspectos que la profesión contable debe abordar, en especial referidos a la información a incluir y su estructura (Davis y Searcy, 2010; Fifka, 2012; Higgins y Walker, 2012).

\section{REFERENCIAS BIBLIOGRÁFICAS}

Ahmad,N.;Sulaiman, M. y Siswantoro, D. (2003),Corporate Social Responsibility in Malaysia: An Analysis of Annual Reports of KLSE listed Companies, IIUM Journal of Economics and Management, v.11, $\mathrm{n}^{\mathrm{o}} 1$, pp. 1-37.

Andonegi, X. A. (2015). Crisis y nueva dirección bancaria (Vol. 58). Universidad de Deusto.

Brammer, S. y Pavelin, S. (2004), Voluntary social disclosures by large UK companies, Business Ethics: A European Review, V. 13, n 2-3, pp. 86-99.

Castka, P., Balzarova, M. A., Bamber, C. J., \& Sharp, J. M. (2004). How can SMEs effectively implement the CSR agenda? A UK case study perspective.Corporate Social Responsibility and Environmental Management, 11(3), 140-149.

Crane, A., Matten, D., \& Spence, L. J. (Eds.). (2008). Corporate social responsibility: Readings and cases in a global context (pp. 5-37). London: Routledge.

Davis, G., \& Searcy, C. (2010). A review of Canadian corporate sustainable development reports. Journal of Global Responsibility, 1(2), 316-329.

Fifka, M. (2012). The development and state of re- search on social and environmental reporting in global comparison. Journal für Betriebswirtschaft, 62(1), 45-84.

Font, X.; Walmsley, A.; Cogotti, S.; Mccombes, L. y Häusler, N., (2012), Corporate social responsibility: The disclosure-performance gap, Tourism Management, v. 33, pp. 1544-1553.

Gray, R., Owen, D. y Adams, C.A. (1996). Accounting and Accountability: changes and challenges in corporate social and environmental reporting. Prentice Halls, Londres.

GRI (2006), Sustainability Reporting Guidelines Version 3.1 (G3), Global Reporting Initiative, Amsterdam.

GRI (2013). G4 Sustainability Reporting Guidelines. Reporting Principles and Standard Disclosures, Global Reporting Initiative, Amsterdam.

Guthrie, J. y Parker, L.D. (1990). Corporate social disclosure practice: a comparative international analysis. Advances in public interest accounting, (3), 159-173.

Hauque S., Di Russo L., del Barco J.P., del Barco M.A. (2016). Responsabilidad Social Empresaria: Una mirada axiológica. Ediciones UNL.

Higgins, C., \& Walker, R. (2012). Ethos, logos, pathos: Strategies of persuasion in social/environmental reports. In Accounting Forum (Vol. 36, No. 3, pp. 194-208). Elsevier.

Ioannou, I., \& Serafeim, G. (2012). What drives corporate social performance? The role of nation-level institutions. Journal of International Business Studies, 43(9), 834-864.

Kabir, M. (2011), Corporate social responsibility by Swaziland hotel industry, Procedia - Social and Behavioral Sciences, v. 25, pp. 73-79.

Krippendorff, K. (1990). Metodología de análisis de contenido. Teoría y Práctica. Piados Comunicación, Barcelona.

Lindorff, M.; Jonson, E. y McGuire, L. (2012), Strategic Corporate Social Responsibility in Controversial Industry Sectors: The Social Value of Harm Minimization, Journal Business Ethics, v. 110, $\mathrm{n}^{\circ}$ 4, pp. 457-467. 
Perrini, F., \& Tencati, A. (2008). Corporate social responsibility: Un nuovo approccio strategico alla gestione d'impresa. Milano: Egea.

Perrini, F. (2006). SMEs and CSR theory: evidence and implications from an Italian perspective. Journal of Business Ethics, 67(3), 305-316.

Rabasedas, ML; del Barco, MA; Jara, LA; del Barco, JP y Hauque, SM (2016). Análisis intersectorial de las memorias de sostenibilidad publicadas por empresas cotizadas en Argentina. Trabajo presentado en el X Simposio de Contabilidad y Auditoría del Extremo Sur. Ushuaia, Argentina.

Salzmann, O., Ionescu-Somers, A., and Steger, U. 2005. 'The Business Case for Corporate Sustainability: Literature Review and Options'. European Management Journal, 23(1): 27-36.

Schadewitz, H. and Niskala, M. (2010), Communication via Responsibility Reporting and its Effect on Firm Value in Finland, Corporate Social Responsibility and Environmental Management, 17(2), 96-106.

Simnett, R., Vanstraelen, A., \& Chua, W. F. (2009). Assurance on sustainability reports: An international comparison. The Accounting Review, 84(3), 937-967.

Thompson J.K., Smith H. L. (1991). Social responsibility and small business: suggestions for research, Journal of Small Business Management, n. 29.

Vives \& Peinado-Vara, E. (2011). La responsabilidad social de la empresa en América Latina. Inter-American Development Bank.

Vives, A., Corral, A., \& Isusi, I. (2006). Responsabilidad Social de la Empresa en las PyMEs de Latinoamérica. Inter-American Development Bank; IKEI.

Williamson, D., Lynch-Wood, G. and Ramsay, J. (2006). Drivers of Environmental Behaviour in Manufacturing SMEs and the Implications for CSR, Journal of Business Ethics, 67, pp. 317-330. 